\title{
Extracorporeal Shock Wave Therapy as Therapeutic Intervention: a Narrative Review
}

\author{
Young-Han Park, Jung-Ho Lee
}

\begin{abstract}
Extracorporeal shock wave therapy has since been developed and used steadily in medical advanced countries. Shock waves have features such as reflection, transmission, and diffraction, as do sound and light waves. Compared to sound waves, they are faster and more powerful pressure waves. ESWT is classified into focus type and radial type, according to the principle of wave generation. Extracorporeal shock wave therapy has been used for a variety of musculoskeletal disorders. Extracorporeal shockwave therapy is a non-surgical and non-invasive treatment. Also, it is applied relatively easily to musculoskeletal patients and it has less impact on daily life since the recovery time after treatment is short.
\end{abstract}

Index Terms- Shock wave, Treatment, Indication, Contraindication.

\section{INTRODUCTION}

Extracorporeal shockwave therapy (ESWT) is a new type of therapy that is exploding in demand as the clinical effect is confirmed for diseases in the field of degenerative osteoarthritis, which is difficult to treat [1]. Shock waves generated in vitro can be focused on specific parts of the human body to treat not only neurological diseases but also orthopedic musculoskeletal diseases [2]. In addition, ESWT has received much attention as an alternative therapy for musculoskeletal disorders and pain treatments, as clinical trial and medical reports indicate that it is effective for the treatment of pain that was incurable with previous conservative treatment methods or surgery. Many studies on ESWT have been actively conducted because it is not only non-invasive and relatively safe, but also effective [3].

Extracorporeal shockwave therapy derived from extracorporeal shockwave lithotripsy, which was first introduced in Germany in 1980 to crush kidney stones using non-invasive shock waves without incision [4]. Afterwards, ESWT devices, which produce relatively lower energy than external lithotripsy focused shock waves, and repeatedly irradiate to treat affected areas of musculoskeletal disorders, were developed in Germany, Switzerland, and Austria in the 1990s [5]. Extracorporeal shockwave therapy has since been developed and used steadily in medical advanced countries because it is a non-invasive and non-surgical therapy that can treat conditions in a short time without anesthesia [6].

Young-Han Park, Department of Physical Therapy, Korea National University of Transportation, Jeungpyeong-gun, Republic of Korea

corresponding author), Department of Physical Therapy, School of Medical \& Public Health, Kyungdong University, Republic of Korea

\section{Study Method}

\section{A. Shock wave generation method}

A shock wave is a large-amplitude sound wave, and a natural shock wave may be generated during an earthquake or thunder, or even during a physical shock, such as a bullet explosion or a collapse of a bubble inside a liquid. It is characterized by delivering great pressure energy in a short time at a supersonic speed [7]. When the shock wave is irradiated to the boundary of the bone/soft tissue, the expansion wave is transmitted into the bone and the pressure wave is reflected to the soft tissue. Shock waves have features such as reflection, transmission, and diffraction, as do sound and light waves. Compared to sound waves, they are faster and more powerful pressure waves. Unlike light, they produce a pressure transducing action that causes the movement of surrounding fluids or solids [8]. In ESWT, a relatively small tensile wave factor is represented as one major positive pressure wave, which has a frequency range of several $\mathrm{kHz}$ to over $10 \mathrm{MHz}$. The focal area of the ESWT is the location on which to focus the shock wave during treatment, and it is the point where $80 \%$ of the maximum emission energy is reached. In this focal area, energy is defined as the energy flux density per impulse, and the unit of the area is recorded in joules [9].

ESWT is classified into focus type and radial type, according to the principle of wave generation. Focus type collects shock waves from the site at which the shock waves are generated through the dust collecting plate. Radial type is a pneumatic radial that uses waves generated by compressed air created with a pendulum of air. Focus type can make strong shock waves as the waves are gathered at one point, while the radial type shock waves weaken as the distance increases due to the spreading shape $[10,11]$.

Focus type converges individual shock waves, which are created by sporadically arranged piezoelectric elements on a conical collector plate inside the shock wave, into one point. This focused shock wave generates a pressure wave of 10 to 100 atmospheres, converts it into a form of vibration energy, and concentrates the energy at a point [12].

The shock wave generators currently used in intensive ESWT equipment are classified into three types: electrohydraulic type, which is the most commonly used method; electromagnetic type; and piezoelectric type. The electrohydraulic shock wave therapy device used in the medical field generates shock waves with electrical sparks between the electrodes caused by high voltage to pointed electrodes facing each other in water at short distances [13]. The electrohydraulic extracorporeal shock wave therapy 
device has to be replaced frequently to maintain high voltage sparks to the shock wave. Also, the increase in operator fatigue due to the noise of continuously generated shock waves is a concern. Thus, there is a need for improvements in the durability of electrodes and the working environment regarding workplace noise [14].

The electroconductive type of shock wave, one generation method in the ESWT device, uses a method of encapsulating the electrode and injecting a self-made electrolyte inside to extend the duration of its life. Such a method increases the cost of the expendables that need to be replaced, thereby increasing the burden of the patient's medical expenses [15]. The electromagnetic method can generate a stable shock wave, enhancing the clinical utility. The electromagnetic method generates a magnetic field in the planar coil by current shock and generates an opposite magnetic field in the adjacent insulated metal diaphragm by electrical induction. These magnetic forces cause the metal plate to vibrate, creating shock waves. In the piezoelectric element method, a shock wave is generated in the surrounding fluid by expansion and contraction of a crystalline material made of piezoelectric elements when high-pressure electricity is applied [16].

Radial Shock Wave Therapy (RSWT) generates pulses by a heavy projectile. Radial extracorporeal shock waves radiate energy by the pressure waves. Pressure waves are generated when the condensed air in the pneumatic device is instantaneously released and collides with numerous pendulums arranged in a straight line [17]. The time of increase in the pulse of the RSWT shock wave is relatively longer than that of other ESWT shock waves. Also, the feeling of the pulse is soft, as it has a gentle curve, enabling the patients to receive high energy treatment comfortably. Moreover, RSWT has a shock wave intensity that does not cause cavitation of cells, minimizing damage to tissues and cells in the human body [18].

\section{B. Indications for extracorporeal shockwave therapy}

Extracorporeal shock wave therapy has been used for a variety of musculoskeletal disorders. In particular, it has been proven to be effective in plantar fasciitis of the proximal heel, lateral epicondylitis of the elbow, calcific tendinitis of the shoulder, and nonunion of fracture. In recent years, it has been extended to the treatment of chronic skin ulcers, osteovascular diseases, and myocardial ischemia [19-21]. Treatment time (session) is the basic treatment principle of ESWT. Typically, 3 5 shock wave treatments are required for tendon disorders and tendon attachment portion lesions, and 6 8 shock wave treatments for treating myofascial pain syndrome [22]. In addition, the dosage during the ESWT varies individually based on the degree of pain. Therefore, it is important to adjust the appropriate dosage according to each patient and indications. Starting with low energy (bar or $\mathrm{mJ} / \mathrm{mm}^{2}$ ) at the most painful area, the dose can be gradually increased after careful examination during the treatment [23].

Extracorporeal shockwave therapy has been proven to induce lymph angiogenesis by promoting angiogenesis and up-regulation of vascular endothelial growth factor and basic fibroblast growth factor in a previous study [24]. Subsequently, the effect of the ESWT was confirmed in the study of patients with secondary lymphedema. Therefore, ESWT is recommended as a non-invasive alternative treatment in lymphedema patients [25]. Extracorporeal shock wave treatment improves cell permeability by the mechanical force generated by acoustic shock waves and increases the manifestation of factors involved in lymph vessel formation. It has also been found to improve the elasticity of the skin and promote the organization of skin structure [26].

Extracorporeal shock wave treatment removes the fundamental cause of joint pain through stimulation-free surgery to repair damaged tissue by delivering effective shock waves to degenerative lesions of the musculoskeletal system, tendon rupture caused by excessive exercise, and lime around joints [27]. The extracorporeal shock wave treatment device can achieve the same effect as extracorporeal shock wave lithotripters on calcification tendinitis [28]. A cell-level cavitation caused by the pressure of the shock wave creates bubbles which explode and generate healing material with the physical effect of breaking lime sedimentation components by shock wave stimulation. Treatment is achieved through the biological effects of promoting resorption of sediment that has been cleaved into and accelerating regeneration by inducing angiogenesis [29].

Extracorporeal shockwave therapy is a non-surgical and non-invasive treatment. Also, it is applied relatively easily to musculoskeletal patients and it has less impact on daily life since the recovery time after treatment is short [30]. Furthermore, ESWT is reported to be an effective therapeutic device not only for patients with musculoskeletal disorders but also for those with dystonia, pressure sores, lymphedema, and erectile dysfunction [31].

According to previous study on the effect of ESWT, repeated micro-stimulation through shockwave therapy was effective in relieving pain [32]. Additionally, another study suggested that the therapeutic effect is caused by physical action to heal ruptured tendons, which is led by the change in cell membrane permeability and induction of radical diffusion [33]. In gateway control theory, A beta nerve fibers, which have a relatively fast conduction rate, are rapidly depolarized, whereas depolarization of A delta nerve fibers and $\mathrm{C}$ nerve fibers are suppressed, preventing the delivery of pain. ESWT contributes to early analgesic effects by reducing pain receptors' ability to deliver pain due to the deformation or increase in cell membrane permeability caused by shock waves [34].

In previous studies, ESWT uses the principle of breaking stones and gallstones, causing increased blood flow and blood vessel remodeling, and stimulating and reactivating the healing process of the tendon and surrounding tissues and bones. Therefore, it has been reported to reduce pain by eliminating and stabilizing inflammation [35].

According to a study on the effect of radial extracorporeal shock waves on upper extremity function in patients with rotator cuff calcification tendonitis, there was a significant improvement in the function of the shoulder joint (with CMS scale) after the treatment [36]. Likewise, another study on the effect of intensive extracorporeal shock wave on upper extremity function in patients with rotator cuff calcification has shown the same result as radial extracorporeal shock 
wave [37].

Usage of radial shock waves for treating myofascial pain syndrome occurring in skeletal muscle is gradually increasing. Radial shock waves are suitable for smoothing muscle tissue, for relaxing muscle tension, for locating and treating superficial pain-causing points, and for activating connective tissue for a wide range of treatments [38]. As extracorporeal shock wave treatment is applied to myofascial pain syndrome, the mechanical energy of the shock wave is converted into chemical energy in connective tissue located in the extracellular matrix. With the help of cell membrane receptors containing proteins and ion channels, this energy is conducted to the cell nucleus through the cytoskeleton. Inside the cell nucleus, chain-like signals induce gene transcription and expression, resulting in mechanosensitive kinase and enriching collagenase [39].

In the study of ESWT in patients with cerebral palsy, it was reported that the effect lasted for up to four weeks when stimulation of $0.030 \mathrm{~mJ} / \mathrm{mm}^{2}$ for 1500 times in the soleus muscle and medial and lateral gastrocnemius was performed to pediatric patients once a week [40]. In cerebral palsy patients with extremely stiff upper and lower muscles, the effect of treatment with 2000 times of stimulation in $0.10 \mathrm{~mJ} / \mathrm{mm}^{2}$ for three times a week lasted for two months; however, the effect of treatment was returned to pre-treatment levels after three months, suggesting that additional extracorporeal shock waves may be necessary after three months of previous treatment [41].

\section{Contraindications for extracorporeal shockwave therapy}

Generally, ESWT is prohibited to people with coagulation disorders or those who take anticoagulants or pregnant women. Also, treatment cannot be given to children under 18 years old and cannot be applied to bone growth plate where epiphysis and bone have not grown. In addition, treatment is prohibited for patients with blood clotting disorders or those who are taking medications to prevent blood clotting disorders, patients with rheumatism, diabetes, osteoporosis, and heart pace makers [42]. Treatment with spinal nerves is also considered contraindicated though it is not reported experimentally.

\section{ACKNOWLEDGMENT}

This study was supported by Korea National University of Transportation in 2019.

\section{REFERENCES}

[1] Q. Ji, P. Wang, C. He, "Extracorporeal shockwave therapy as a novel and potential treatment for degenerative cartilage and bone disease: Osteoarthritis. A qualitative analysis of the literature", Prog. Biophys. Mol. Biol., vol 121, 2016, pp. 255-265.

[2] A. A. Divani, P. Salazar, M. Monga, G. J. Beilman, K. S. SantaCruz, "Inducing different brain injury levels using shock wave lithotripsy", J. Am. Inst. Ultrasound Med., vol 37, 2018, pp. 2925-2933.

[3] V. Korakakis, R. Whiteley, A. Tzavara, N. Malliaropoulos, "The effectiveness of extracorporeal shockwave therapy in common lower limb conditions: a systematic review including quantification of patient-rated pain reduction", Br. J. Sports Med., vol 52, 2018, pp. $387-407$.

[4] C. Chaussy, G. Fuchs, "Experience with extracorporeal shockwave lithotripsy based on 5 years' clinical use", Urol. Ausg A, vol 24, 1985 pp. 305-309.
[5] Z. Lu, G. Lin, A. Reed-Maldonado, C. Wang, Y.-C. Lee, T. F. Lue, "Low-intensity extracorporeal shock wave treatment improves erectile function: a systematic review and meta-analysis", Eur. Urol., vol 71, 2017, pp. 223-233.

[6] S. E. G. Sims, K. Miller, J. C. Elfar, W. C. Hammert, "Non-surgical treatment of lateral epicondylitis: a systematic review of randomized controlled trials", Hand N. Y. N, vol 9, 2014, pp. 419-446.

[7] J. D. Rompe, J. Furia, A. Cacchio, C. Schmitz, N. Maffulli, "Radial shock wave treatment alone is less efficient than radial shock wave treatment combined with tissue-specific plantar fascia-stretching in patients with chronic plantar heel pain", Int. J. Surg. Lond. Engl., vol 24, 2015, pp. 135-142.

[8] S. W. Ohl, E. Klaseboer, A. J. Szeri, B. C. Khoo, "Lithotripter shock wave interaction with a bubble near various biomaterials", Phys. Med. Biol., vol 61, 2016, pp. 7031-7053.

[9] N. Sheveleva, L. Minbayeva, Y. Belyayeva, "Shock-wave therapy application in clinical practice (review)", Georgian Med. News, vol 3, 2016, pp. 42-47.

[10] S. Sundaram et al., "Stimulation of angiogenesis using single-pulse low-pressure shock wave treatment", J. Mol. Med. Berl. Ger., vol 96, 2018, pp. 1177-1187.

[11] L. A. Khodyreva et al., "Efficiency assessment of shock wave therapy in patients with pelvic pain employing harmonic analysis of penile bioimpedance", Bull. Exp. Biol. Med., vol 155, 2013, pp. 288-292.

[12] M. J. Choi, G. Kang, J. S. Huh, "Geometrical characterization of the cavitation bubble clouds produced by a clinical shock wave device", Biomed. Eng. Lett., vol 7, 2017, pp. 143-151.

[13] E. Özkan, M. C. Bereket, E. Şenel, M. E. Önger, "Effect of electrohydraulic extracorporeal shockwave therapy on the repair of bone defects grafted with particulate allografts", J. Craniofac. Surg., vol 30, 2019, pp. 1298-1302.

[14] C. J. Lischer et al., "Treatment of chronic proximal suspensory desmitis in horses using focused electrohydraulic shockwave therapy", Schweiz. Arch. Tierheilkd., vol 148, 2006, pp. 561-568.

[15] T. Flam et al., "Electroconductive extracorporeal lithotripsy with sonolith 4000+. a prospective study of the ECG desynchronization using a fixed-rhythm simulator", J. Urol. (Paris), vol 102, 1996, pp. 195-198.

[16] N. Tomska et al., "Deep electromagnetic stimulation and radial shock wave therapy in back pain", Ortop. Traumatol. Rehabil., vol 20, 2018, pp. 189-195.

[17] B. Akinoglu, N. Köse, N. Kirdi, Y. Yakut, "Comparison of the acute effect of radial shock wave therapy and ultrasound therapy in the treatment of plantar fasciitis: a randomized controlled study", Pain Med. Malden Mass, vol 18, 2017, pp. 2443-2452.

[18] M. I. Ibrahim, R. A. Donatelli, M. Hellman, A. Z. Hussein, J. P. Furia, C. Schmitz, "Long-term results of radial extracorporeal shock wave treatment for chronic plantar fasciopathy: A prospective, randomized, placebo-controlled trial with two years follow-up", J. Orthop. Res. Off. Publ. Orthop. Res. Soc., vol 35, 2017, pp. 1532-1538.

[19] J. Lou, S. Wang, S. Liu, G. Xing, "Effectiveness of extracorporeal shock wave therapy without local anesthesia in patients with recalcitrant plantar fasciitis: a meta-analysis of randomized controlled trials", Am. J. Phys. Med. Rehabil., vol 96, 2017, pp. 529-534.

[20] R. R. Bannuru, N. E. Flavin, E. Vaysbrot, W. Harvey, T. McAlindon, "High-energy extracorporeal shock-wave therapy for treating chronic calcific tendinitis of the shoulder: a systematic review", Ann. Intern. Med., vol 160, 2014, pp. 542-549.

[21] N. Haffner, et al., "Extracorporeal shockwave therapy (ESWT) ameliorates healing of tibial fracture non-union unresponsive to conventional therapy", Injury, vol 47, 2016, pp. 1506-1513.

[22] Q. Wang, F. Jiang, "Principle and application of orthopedic ESWT apparatus", Zhongguo Yi Liao Qi Xie Za Zhi, vol 34, 2010, pp. 297-299.

[23] Y. E. Moon, H. Seok, S.-H. Kim, S. Y. Lee, J. H. Yeo, "Extracorporeal shock wave therapy for sacroiliac joint pain: A prospective, randomized, sham-controlled short-term trial", J. Back Musculoskelet. Rehabil., vol 30, 2017, pp. 779-784.

[24] E. Higashihara, M. Fujime, T. Niijima, "Extracorporeal shockwave lithotripsy and lymph node calcification”, Dtsch. Med. Wochenschr. 1946, vol 112, 1987, pp. 763-764.

[25] S.-Y. Kim, H. Bae, H. M. Ji, "Computed tomography as an objective measurement tool for secondary lymphedema treated with extracorporeal shock wave therapy", Ann. Rehabil. Med., vol 39, 2015, pp. $488-493$.

[26] I. G. Kim, J. Y. Lee, D. S. Lee, J. Y. Kwon, J. H. Hwang, "Extracorporeal shock wave therapy combined with vascular endothelial growth factor-C hydrogel for lymphangiogenesis", J. Vasc. Res., vol 50, 2013, pp. 124-133. 
[27] S. Mani-Babu, D. Morrissey, C. Waugh, H. Screen, C. Barton, "The effectiveness of extracorporeal shock wave therapy in lower limb tendinopathy: a systematic review", Am. J. Sports Med., vol 43, 2015 pp. 752-761.

[28] W.-Y. Chou, C.-J. Wang, K.-T. Wu, Y.-J. Yang, J.-Y. Ko, K.-K. Siu, "Prognostic factors for the outcome of extracorporeal shockwave therapy for calcific tendinitis of the shoulder", Bone Jt. J., vol 99-B, 2017, pp 1643-1650.

[29] K. Yahata, et al., "Low-energy extracorporeal shock wave therapy for promotion of vascular endothelial growth factor expression and angiogenesis and improvement of locomotor and sensory functions after spinal cord injury", J. Neurosurg. Spine, vol 25, 2016, pp $745-755$.

[30] J. M. Reilly, E. Bluman, A. S. Tenforde, "Effect of shockwave treatment for management of upper and lower extremity musculoskeletal conditions: a narrative review", PM R, vol 10, 2018, pp. 1385-1403.

[31] S. M. Jeppesen, K. B. Yderstraede, B. S. B. Rasmussen, M. Hanna, L. Lund, "Extracorporeal shockwave therapy in the treatment of chronic diabetic foot ulcers: a prospective randomised trial", J. Wound Care, vol 25, 2016, pp. 641-649.

[32] [32] S. Aktürk, et al., "Comparision of the effectiveness of ESWT and ultrasound treatments in myofascial pain syndrome: randomized, sham-controlled study", J. Phys. Ther. Sci., vol 30, 2018, pp. 448-453.

[33] K. Storheim, L. Gjersing, K. Bølstad, M. A. Risberg, "Extracorporeal shock wave therapy (ESWT) and radial extracorporeal shock wave therapy (rESWT) in chronic musculoskeletal pain", Tidsskr. Den Nor. Laegeforening Tidsskr. Prakt. Med. Ny Raekke, vol 130, 2010, pp. 2360-2364.

[34] T. Klonschinski, S. J. Ament, T. Schlereth, J. D. Rompe, F. Birklein, "Application of local anesthesia inhibits effects of low-energy extracorporeal shock wave treatment (ESWT) on nociceptors", Pain Med. Malden Mass, vol 12, 2011, pp. 1532-1537.

[35] J.-H. Cheng, C.-J. Wang, "Biological mechanism of shockwave in bone", Int. J. Surg. Lond. Engl., vol 24, 2015, pp. 143-146.

[36] F. Ioppolo, et al., "Clinical improvement and resorption of calcifications in calcific tendinitis of the shoulder after shock wave therapy at 6 months' follow-up: a systematic review and meta-analysis", Arch. Phys. Med. Rehabil., vol 94, 2013, pp. 1699-1706.

[37] A. Arirachakaran, M. Boonard, S. Yamaphai, A. Prommahachai, S. Kesprayura, J. Kongtharvonskul, "Extracorporeal shock wave therapy, ultrasound-guided percutaneous lavage, corticosteroid injection and combined treatment for the treatment of rotator cuff calcific tendinopathy: a network meta-analysis of RCTs", Eur. J. Orthop. Surg. Traumatol. Orthop. Traumatol., vol 27, 2017, pp. 381-390.

[38] [38] J. O. Hong, J. S. Park, D. G. Jeon, W. H. Yoon, J. H. Park, "Extracorporeal shock wave therapy versus trigger point injection in the treatment of myofascial pain syndrome in the quadratus lumborum", Ann. Rehabil. Med., vol 41, 2017, pp. 582-588.

[39] J. H. Jeon, et al., "The effect of extracorporeal shock wave therapy on myofascial pain syndrome", Ann. Rehabil. Med., vol 36, 2012, pp. 665-674.

[40] A. Mirea, G. Onose, L. Padure, E. Rosulescu, "Extracorporeal shockwave therapy (ESWT) benefits in spastic children with cerebral palsy (CP)", J. Med. Life, vol 7, 2014, pp. 127-132.

[41] D.-S. Park, D. R. Kwon, G.-Y. Park, M. Y. Lee, "Therapeutic effect of extracorporeal shock wave therapy according to treatment session on gastrocnemius muscle spasticity in children with spastic cerebral palsy: a pilot study", Ann. Rehabil. Med., vol 39, 2015, pp. 914-921.

[42] F. Q. Gao, W. Sun, G. Y. Xing, "The interpretation on ESWT indications and contraindications of international society for medical shockwave treatment", Zhonghua Yi Xue Za Zhi, vol 97, 2017, pp. 2411-2415.
Young-Han Park, he received PhD degree from Daegu University, Republic of Korea. His research interests include Electrical therapy, Phototherapy, Neurophysiology, Neuroanatomy, and Sports Physiotherapy. He had completed a lot of training in orthopedic and neurological Sciences Institute. He is currently working as Professor of Department of Physical Therapy, Korea National University of Transportation, Republic of Korea.

Jung-Ho Lee, $\mathrm{He}$ received $\mathrm{PhD}$ degree from Daegu University, Republic of Korea. His research interests include Shoulder Disorder, Neurophysiology, Neuroanatomy, Ergonomics, Work Physiology and Sports Physiotherapy. He had completed a lot of training in orthopedic and neurological Sciences Institute. He is currently working as Professor of Department of Physical Therapy, Kyungdong University, Republic of Korea. 